\title{
Allium sativum: Oral mucosal burn
}

\author{
Bharati Patil ${ }^{1}$, Ruchika A.N ,** $^{2}$
}

${ }^{1}$ Professor, ${ }^{2} 3^{\text {rd }}$ Year Post Graduate Student, Dept. of Oral Medicine \& Radiology, The Oxford Dental College, Bangalore, Karnataka, India

\author{
*Corresponding Author: Ruchika A.N
}

Email: ruchika.nerurkar879@gmail.com

\begin{abstract}
Introduction: Oral medicine practitioner has reported many cases of chemical burn in the oral mucosa. Inappropriate self-treatment with topical application of various therapeutic or non-therapeutic agents often causes chemical burns. Garlic is a century old herb, commonly used in culinary which can cause oral mucosal burns when misused.

Case Description: A male patient aged 55 years, reported to the department with a history of tooth pain since one month which had increased in intensity 3 days back. To alleviate the dental pain he had applied crushed raw garlic (Allium sativum) at the site of pain for 60 minutes consecutively for 3 days before visiting the hospital. On intra-oral clinical examination, white pseudo membranous lesion surrounded by erythema was noted on right posterior maxillary and mandibular attach gingiva. At the site of crushed raw garlic application, localized tissue necrosis was observed. The patient was advised to stop self-treatment with raw garlic and was prescribed systemic and topical analgesics to relieve the dental pain.
\end{abstract}

Conclusion: This case report highlights the importance of clinical case history recording. The oral physician should be aware of the herbs like garlic which are commonly used to alleviate pain, which can cause adverse effects like mucosal burn if used inappropriately.

Keywords: Chemical burn, garlic, Allium sativum, Oral soft-tissue burns, Mucosal burn.

\section{Introduction}

Oral soft tissue injuries are caused by thermal, chemical and physical agents which results in oral soft-tissue burns.

Oral mucosal burns can be iatrogenic or accidental and are frequently reported cases in the literature. The inappropriate self-treatment with therapeutic medicaments like Aspirin, Chlorpromazine or non-pharmacological agents like garlic, clove to alleviate pain often causes oral soft-tissue burns when the medicament comes in direct contact with the oral mucosa. ${ }^{1-3}$

The extent of the oral mucosal injury due to Chemical depends on the factors like $\mathrm{pH}$, concentration and manner of application of the medicament (acidity or alkalinity), and the duration of tissue contact, the extent of penetration of medicament into the tissue and mechanism of the action of the medicament on the mucosa.,

Allium sativum known as garlic is a bulbous perennial plant known by various names like, ago, Allium, clove garlic which has been used as a flavouring agent, as well as for medicinal purposes for over 5,000 years. It has antimicrobial, anti-oxidant, anti-diabetic and anti-carcinogenic activity is used to treat various diseases since ancient times. ${ }^{5}$

Garlic has a high content of organo-sulfur which consist of g-glutamyl peptides and allylcysteine sulfoxides. A raw crushed, squeezed or cut, bulb of garlic has allylcysteine sulfoxide which metabolizes to allicin acetylcysteine action of alliinase and readily converts into more stable the compounds like polysulfides. Allicin present in garlic prevents proliferation of Helicobacter pylori in stomach. Allicin further breaks down to form a compound called Ajoene which has anticoagulant action. Garlic also contains Carminative which is known to ease the griping pains., ${ }^{1,5,6}$

In this article, we report a case of chemical burn in oral mucosa due to application of topical raw crushed garlic in the right maxillary and mandibular attached gingiva.

\section{Description}

A male patient aged 55 years visited the department with a chief complaint of intermittent, throbbing pain in the right maxillary molar region for past one-month with a history of pain which increased in intensity 3 days back. In an effort to relieve the dental pain he applied a crushed raw garlic at the site of pain for an hour for 3 consecutive days before seeking the dental treatment at the hospital. On the extra-oral examination, no palpable lymph-nodes and facial asymmetry were observed.

The intraoral examination revealed white pseudo membranous lesion with erythema on right maxillary attached gingiva of premolars and first molar region $(14,15$, 16) and right mandibular attach gingiva of premolars and first molar region $(44,45,46)$. The involved area appeared to be soft and tender on palpation. The lesion was scrapable and raw bleeding was observed when the lesion was scraped. Hard tissue examination revealed grade four furcation involvement and grade one mobility in right maxillary first molar (16). Since the first molar (16) had poor prognosis, patient was advised to undergo extraction. The patient was advised to stop self-treatment with raw garlic and was prescribed topical analgesics $(0.15 \%$ benzydamine hydrochloride- to swish and spit thrice a day half an hour before food for 7 days) and systemic analgesic (Ibuprofen$400 \mathrm{mg}$ ) thrice daily for 3 days).

The patient reported three days after the first visit for the follow-up and intra-oral examination revealed regressed lesion with erythema on the attached gingiva. The patient was advised to continue with the analgesics for 5 days.

On second follow-up one week after the initial visit, the regressed lesion with erythema was observed on maxillary attach gingiva. Lesion on mandibular attach gingiva had completely regressed. Patient was advised extraction and was referred to Department of Oral and Maxillofacial Surgery. Post extraction the patient was lost to follow-up. 


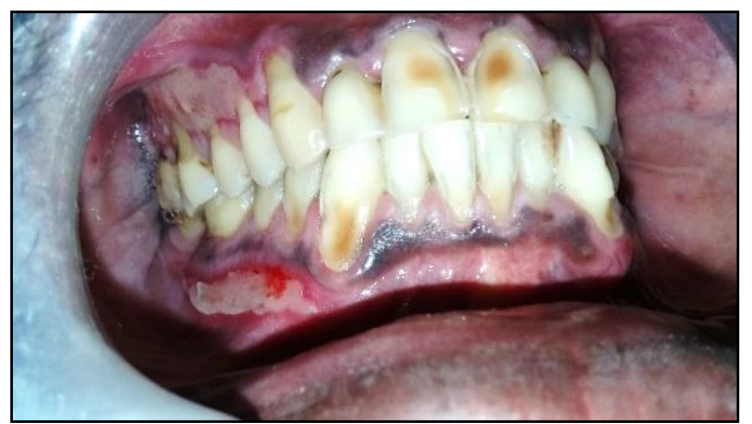

Fig. 1: A White pseudo membranous lesion with erythema present on right maxillary and mandibular first and second premolars and first molar region and raw bleeding region present on the mandibular attach gingiva of first premolar region

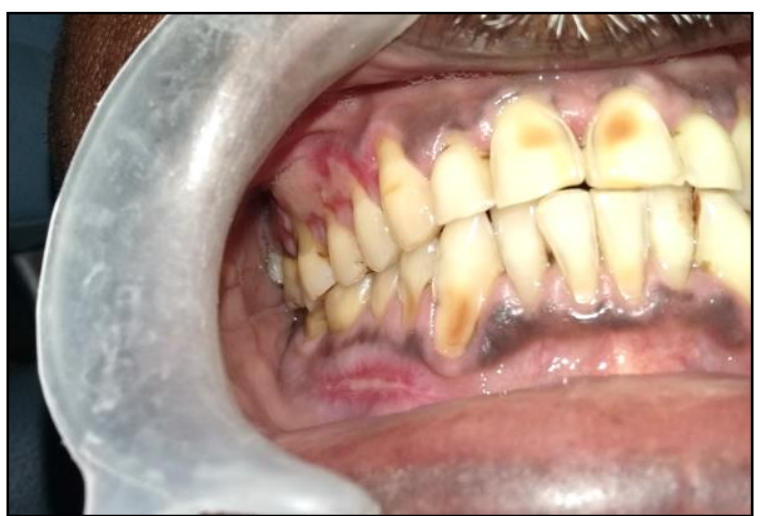

Fig. 2: First follow-up intra-oral examination revealed regressed white pseudo membranous lesion with erythema on right maxillary and mandibular attach gingiva

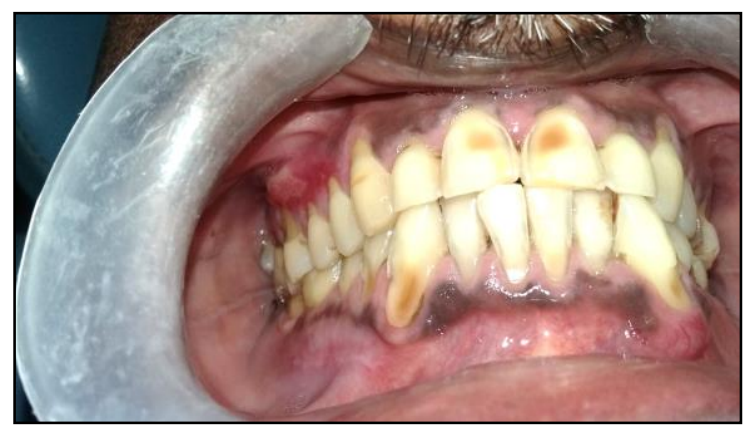

Fig. 3: Second follow-up, white pseudo membranous lesion with erythema was noted on maxillary attach gingiva of the right maxillary premolars and first molar region. Lesion on mandibular attach gingiva had completely regressed

\section{Discussion}

Allium sativum popularly known as garlic is one of the most commonly used spice or herb in the kitchen. Garlic has anti-bacterial, anti-viral, anti-helminthic, anti-diabetic and anti- carcinogenic properties. Garlic has high content of organ sulfur which metabolizes into Allicin an oxidized derivative of diallyl disulfide which is responsible for causing acantholysis. Allicin affects the metabolism of cysteine in proteins causing disruption of the epidermal junction further causing coagulative necrosis of mucosa which results in mucosal burn. ${ }^{5}$

Vargo RJ reported a case of a male patient aged 49-years who reported to the department for the treatment of maxillary tooth pain intra-oral examination revealed chemical burn in oral mucosal region of maxillary vestibule caused due to topical application of crushed raw garlic to alleviate dental pain. Analgesics were prescribed to ease the pain. ${ }^{1}$

Yilmaz HH reported a case of a female patient aged 80years- who was referred to department with severe, lancinating pain which occurred intermittently on the left side of her face for a period of three years and was diagnosed with trigeminal neuralgia. During one such episode of trigeminal neuralgia to relive the pain patient had applied crushed garlic, wrapped in cloth, on left side of her face for an hour and suffered from second- degree chemical burn on her face, which was treated with topical Bepanthene cream. ${ }^{4}$

In our case the patient had tooth pain in right maxillary first molar region which was self- treated by applying raw crushed garlic which caused chemical burn in right maxillary and mandibular attach gingiva, which was treated with benzydamine hydrochloride and systemic analgesics.

\section{Conclusion}

This case report highlights the importance of clinical case history taking. Oral physician should be aware of natural herbs used to alleviate pain in day to day life, which when used inappropriately can cause soft tissue burn.

\section{Conflict of Interest: Nil.}

\section{References}

1. Vargo RJ, Warner BM, Potluri A, Prasad JL. Garlic burn of the oral mucosa: A case report and review of self-treatment chemical burns. J Am Dent Assoc. 2017 Apr 5.

2. Gilvetti C, Porter SR, Fedele S. Traumatic chemical oral ulceration: a case report and review of the literature. Br Dent $J$. 2010;208(7):297-300.

3. Deepanjeet Kaur, Kaushal K Chandrul., Syzygium aromaticum L. (Clove): A vital herbal drug used in periodontal disease. Indian J Pharm Biol Res. 2017;5(2)45-51.

4. Yilmaz HH, Gormez O, Hastar E, Yildirim D, Aksoy MC. Garlic burn in a patient with trigeminal neuralgia: a case report. European journal of dentistry. 2010;4(1):88.

5. Adaki S, Adaki R, Shah K, and Karagir A. Garlic: Review of literature. Indian J Cancer. 2014; 51:577-581.

6. Tyagi S. Importance of Garlic (Allium Sativum): An Exhaustive Review. J Drug Discov Ther. 2013;1(04).

How to cite this article: Patil B, Ruchika A.N. Allium sativum: Oral mucosal burn. Int J Oral Health Dent. 2018;4(4):247-248. 